\title{
Risk factors for ureteroscopic lithotripsy: a case-control study and analysis of 385 cases of holmium laser ureterolithotripsy
}

\author{
Jiaxin Zheng, Yongfeng Wang, Bin Chen, Huiqiang Wang, Rongfu Liu, Bo Duan, Jinchun Xing \\ Department of Urology and Center of Urology, First Affiliated Hospital of Xiamen University, Xiamen, China \\ Videosurgery Miniinv 2020; 15 (1): 185-191 \\ DOI: https://doi.org/10.5114/wiitm.2019.85360
}

\begin{abstract}
Introduction: Ureteroscopic lithotripsy has become the first choice for the treatment of middle and lower ureteral stones, but it still has a certain rate of surgical failure. Here we aimed to determine the factors that may affect the success rate of holmium laser ureterolithotripsy $(H L U)$ and provide the basis and guidance for its future use.

Aim: To evaluate the risk factors for HLU failure.

Material and methods: The clinical data of 385 patients undergoing holmium laser ureterolithotripsy from 2009 to 2012 were retrospectively reviewed to analyze the impact of gender, age, stone side, stone size, stone location, stone number, degree of hydronephrosis, stone impaction, previous extracorporeal shock lithotripsy (ESWL), and associated urinary tract infection (UTI) on the success or failure of surgery.

Results: Surgical success was achieved in 338 (87.8\%) patients versus surgical failure in $47(12.2 \%)$ patients. Univariate analysis revealed that the degree of hydronephrosis $(p=0.024)$, stone impaction $(p=0.003)$, stone location $(p=$ $0.012)$, and previous ESWL ( $p=0.037)$ were risk factors for surgical failure. Multivariate logistic regression revealed that stone impaction (odds ratio $(O R)=2.66 ; p=0.018$ ) and stone location $(O R=2.11 ; p=0.013$ ) were significantly associated with surgical failure. Since some cases of ureterostenosis developed postoperatively, we continued follow-up. The patients had the stent for a year and underwent regular follow-up checks until 5 years. No cases of ureterostenosis recurred.

Conclusions: Ureteroscopic lithotripsy is a safe procedure with few complications. Stone impaction and proximal location are the risk factors for its failure.
\end{abstract}

Key words: complication, lithotripsy, ureteral stone, ureteroscopy.

\section{Introduction}

Due to the effects of the increasing incidence of obesity, diabetes, and changes in dietary habits, ureteral stones have become more common [1, 2]. Without treatment, it may lead to chronic kidney disease [3] and renal failure [4]. The treatment of ureteral stones varies from the management of spontaneous passage to the use of refined endourologic techniques and minimally invasive procedures that can provide a less painful and faster recovery [5].
Ureteroscopic lithotripsy (URL) has become the first choice for the treatment of middle and lower ureteral stones due to its safety and effectiveness [6]. Short-interval shock wave lithotripsy sessions are safe and effective for treating upper ureteral stones [7]. Because of its precision and strong decomposing power, the holmium laser has become one of the most popular tools in urological procedures, including lithotripsy $[8,9]$. One major advantage of the holmium laser is that it can efficiently fragmentize urinary calculi regardless of their size, 
hardness, chemical composition, or physical consistency; accordingly, a high stone-free rate can be achieved $[10,11]$. Hence, it is recommended as the gold standard for intracorporeal lithotripsy for the endoscopic management of urinary tract stones [12, 13]. Although its safety and effectiveness have gradually improved, it still has a certain rate of surgical failure [14].

\section{Aim}

This study aimed to explore the risk factors for holmium laser ureterolithotripsy (HLU) failure. Since the composition, size, and impaction of kidney stones differ among Chinese, European, and American patients and this technology arrived in China only recently, here we aimed to determine the factors that may affect the success rate of HLU and provide the basis and guidance for its future use.

\section{Material and methods}

\section{General information}

A total of 385 patients with ureteral stones treated with HLU at our hospital between 2009 and 2012 were selected for retrospective analysis. Inclusion criteria were as follows: age, 15-80 years; diagnosed by preoperative intravenous pyelography or computed tomography; and no history of lithotripsy. Exclusion criteria were complication of ureteral polyp or ureteral stricture, ureteral stent, malignant tumor, other diseases contraindicated for URL. This study was conducted in accordance with the Declaration of Helsinki. This study was conducted with approval from the Ethics Committee of Xiamen University. Written informed consent was obtained from all participants. The patients were divided into two groups according to the surgical results: the success group (S) and the failure group (F). Patients were assigned to the $S$ group if the stones were completely exhausted within 1 month after HLU and no complications occurred within 3 months and to the $F$ group if either incomplete stone drainage, requirement of other lithotripsy methods (ESWL, ureteral lithotripsy, or open surgery) for resolution, or the occurrence of certain complications was noted. The impact of factors such as sex, age, stone side, size, number, and impaction (stone stopped anywhere within the ureter and could not be excreted from the body), degree of hydronephrosis, history of extracorpore- al lithotripsy, and associated urinary tract infection were analyzed. Ultrasonographic examination of the renal pelvis at the extended service life of $10 \mathrm{~mm}$ or with the presence of renal calyces is called hydronephrosis. The type of hydronephrosis was classified as below: (1) mild hydronephrosis: renal morphology and size were mostly without obvious abnormalities in renal parenchymal thickness and echo normal renal collection system separation of $2-3 \mathrm{~cm}$; (2) moderate hydronephrosis: the renal volume slightly increased, the shape was plump, the parenchyma was slightly thinner, and the renal column appeared unclear. The pelvis and calyces were all significantly dilated, and the renal collecting system was separated by $3-4 \mathrm{~cm}$; (3) severe hydronephrosis: the renal volume increases and the morphologic aberrant parenchyma thins significantly or the entire renal area cannot be seen as a dark fluid area.

\section{Treatment}

All procedures were performed by the same team. After continuous epidural anesthesia or general anesthesia was induced, each patient was placed in the lithotomy position. A Wolf Fr8.0/9.8 rigid ureteroscope and medical holmium laser apparatus (Lumenis Co, CA, USA.) were used. The ureteroscope was inserted into the bladder through the urethra under direct vision, during which period the visual field was kept clear. After reaching the stone site, a $365-\mu \mathrm{m}$ holmium optical laser fiber was inserted, which broke the stone to pieces $<2 \mathrm{~mm}$. In patients with stenosis, we used a ureteroscope body for direct dilation or holmium laser incision. If a patient suffered from moderate to severe hydronephrosis before surgery or had stones adhering to the ureter wall under endoscopy, which had to be detached from the wall, stone impaction could be determined [15]. Postoperatively, the ureters were routinely indwelled by a double $J$ tube for 1-3 months and the bladder was catheterized for 1-3 days. Each patient's condition was closely observed after surgery. A kidney, ureter, and bladder plain film was collected on the first postoperative day to define the position of the double J tube and the surgical effects. Computed tomography was performed 1 month postoperatively to determine the stone clearance. Intravenous urography was performed 3 months postoperatively, and the patients were followed up for 3-60 months (mean: 12 months). 


\section{Statistical analysis}

SPSS ver. 15.0 statistical software (SPSS Inc., Chicago, IL, USA) was used for all statistical analyses. The univariate analysis was performed using the $\chi^{2}$ test and $t$-test. The multivariate analysis was performed to determine the risk factors by logistic regression. $P<0.05$ was considered to indicate statistical significance.

\section{Results}

This study included 385 patients (257 males, 128 females aged 15-80 years, mean: 44.4 years). The disease duration of the 385 patients ranged from 1 day to 3 years (mean: 2.3 months). The stone size was $0.6-2.2 \mathrm{~cm}$ (mean: $0.93 \mathrm{~cm}$ ). There were 91 cases of upper stones and 294 cases of middle and lower stones. Preoperative urine culture exhibited positive results in 52 cases, and all patients underwent surgery once the infection was resolved. A total of 45 patients with concomitant diabetes underwent surgery after having achieved stable blood glucose control. The operation was completed without fluoroscopy but was guided by a guide wire. In the process of lithotripsy, the larger stones were clamped out with ureteroscopic forceps. Meanwhile, the stones were smashed into pieces $<3 \mathrm{~mm}$ whenever possible, and a thinner ureteral stent was retained to avoid obstruction. All the operations were performed by the same chief physician and his attending physician. The chief and attending physicians have performed more than 800 surgical procedures. Of our cohort, surgery was successful in 338 (87.8\%) and failed in $47(12.2 \%)$ cases. Among the 47 failure cases, 17 were due to the stone migrating into the renal pelvis during surgery, preventing lithoclasty, and the surgical method was changed to flexible URL or indwelling double J tube for ESWL. Thirteen cases failed due to ureteral twist, stenosis, or polyp formation below the stone that blocked direct vision of the stone and required conversion to URL. Five cases failed due to ureter perforation during lithotripsy, among which 2 were treated with postoperative stenting and 3 were changed to open surgery. Three cases failed due to the larger ureter angle caused by the ureteroscopy failure; in such cases, double J tubes were placed in the ureter for ESWL. Postoperative complications occurred in 9 patients, including 6 with a fever greater than $38.5^{\circ} \mathrm{C}$ (among whom 4 had positive preoperative urine cultures) and 3 with ureteral ste- nosis 3 months postoperatively. All of these patients had stone impaction and underwent HLU and stent placement for 1 year. Since some patients developed ureterostenosis postoperatively, we continued to follow them up with regular checks for 5 years. No cases of ureterostenosis recurrence occurred.

Univariate analysis showed statistically significant differences in the degree of hydronephrosis, stone impaction, stone site, and history of preoperative or extracorporeal lithotripsy between the two groups ( $p<0.05)$ (Tables I and II). Multivariate logistic regression analysis showed that stone impaction and stone site were the risk factors for surgical failure (Table III).

\section{Discussion}

We found that the most important risk factors leading to surgical failure were stone impaction and upper ureteral calculi. In this study, the failure rate of patients with stone impaction was 20.6\% (21/102) versus $9.2 \%(26 / 283)$ in patients without stone impaction. The URL is the preferred treatment for middle and lower ureteral calculi [16]. When treating certain cases with upper ureteral stone impaction, HLU also has unique advantages [17]. In recent years, due to the improvement of surgical equipment, advances in surgical techniques, and strict selection of indications, the success rate of such surgery has been significantly improved. The total success rate reported in the literature is $81-94 \%$ [18], which is significantly better than ESWL. The overall complication rate is $4.7-9 \%[19,20]$, while the incidence rate of perforation is less than $2 \%$ [21]. Some scholars have shown that the efficacy of ureteroscopic surgery using a Fr7.5 is significantly better than that using a Fr10 [22].

However, due to the long and curved ureters, small lumens, 3 cases of physiological stenosis, and rigidity or semi-rigidity of most ureteroscopes, new surgeons may encounter certain difficulties when performing such surgery, which may cause complications that lead to URL failure. The main reasons for URL failure include ureteroscope placement failure; lithotripsy failure; ureteral perforation; intraoperative and postoperative ureteral hemorrhage; ureteral mucosal avulsion; or postoperative high fever.

Stone impaction is prone to polyp formation since the stones may cause long-term irritation of the ureteral wall, thus causing mucosal edema or 
Table I. Univariate analysis of risk factors for failure in ureteroscopic lithotripsy (categorical variables)

\begin{tabular}{|c|c|c|c|}
\hline Factor & Total cases $(n=385)$ & Failures, $n$ (\%) & $P$-value \\
\hline Gender: & & & 0.063 \\
\hline Male & 257 & $37(4.4)$ & \\
\hline Female & 128 & $10(7.8)$ & \\
\hline Side: & & & 0.682 \\
\hline Left & 194 & $25(12.9)$ & \\
\hline Right & 191 & $22(11.5)$ & \\
\hline Hydronephrosis: & & & 0.024 \\
\hline No or mild & 289 & $29(10.0)$ & \\
\hline Medium and heavy & 96 & $18(18.8)$ & \\
\hline Stone impaction: & & & 0.003 \\
\hline Yes & 102 & $21(20.6)$ & \\
\hline No & 283 & $26(9.2)$ & \\
\hline Stone site: & & & 0.012 \\
\hline Upper ureter & 91 & $18(19.7)$ & \\
\hline Middle and lower ureter & 294 & $29(9.9)$ & \\
\hline Number of stones: & & & 0.501 \\
\hline Single & 309 & $36(11.7)$ & \\
\hline Multiple & 76 & $11(14.5)$ & \\
\hline Preoperative urinary tract infection: & & & 0.978 \\
\hline Yes & 65 & $8(12.3)$ & \\
\hline No & 320 & 39 (12.1) & \\
\hline Preoperative history of ESWLL: & & & 0.037 \\
\hline Yes & 121 & $21(17.4)$ & \\
\hline No & 264 & $26(9.8)$ & \\
\hline
\end{tabular}

Table II. Univariate analysis of risk factors for failure in ureteroscopic lithotripsy (continuous variables)

\begin{tabular}{|lccc|}
\hline Factor & Mean in group S & Mean in group F & $P$-value \\
\hline Age & $44.2 \pm 10.1$ & $46.2 \pm 12.4$ & 0.218 \\
\hline Stone size & $9.2 \pm 5.3$ & $10.2 \pm 6.3$ & 0.238 \\
\hline
\end{tabular}

Table III. Multivariate logistic regression analysis of risk factors for failure in ureteroscopic lithotripsy

\begin{tabular}{|lccc|}
\hline Risk factors & $P$-value & OR & $95 \% \mathrm{Cl}$ \\
\hline Stone site & 0.018 & 2.11 & $1.11-3.74$ \\
\hline Stone impaction & 0.013 & 2.66 & $1.22-5.61$ \\
\hline
\end{tabular}


inflammation that in turn results in polyps, granulation tissues, and even ureteral lumen obstruction in severe cases that prevents stone visualization and may lead to surgical failure. If such patients are complicated with more polyps, long-term stone impaction or highly mucosal edema and fragility, the safety guide wire may easily pass through the mucosa, resulting in submucosal false passage or perforation. Together with inaccurate operations, the fragile polyps in such injured tissues or mucous membranes can cause bleeding. Unclear vision can also lead to surgical failure [23]. An intraoperative ureteral mucosal injury can easily lead to postoperative ureteral stenosis. The 3 cases of ureteral stenosis 3 months postoperatively were those with stone impaction. Intracorporeal lithotripsy during the ureteroscopic management of impacted ureteral stones is highly efficient with high success rates regardless of stone location [24]. At the same time, the recurrence rate of postoperative stones was also higher than that of the open surgery group (13.1\% to $4 \%)$. The author believes that when managing stones in patients with concomitant ureteral impairment, removal of the diseased ureter can be more therapeutic. Therefore, for patients with stone impaction, the stones should be pushed up until the ureter is dilated as much as possible during surgery. It is best not to treat the stones in situ. Due to local inflammatory reactions, such stones easily cause injury and postoperative ureteral stenosis. Surgeons should try to completely fragment the stones and avoid repeatedly encasing or clamping them. At the same time, long-term follow-up after ureteral lithotomy is very important since it can identify long-term complications that require prompt treatment, such as ureteral stenosis.

In addition, the univariate analysis showed a statistically significantly difference in the degree of hydronephrosis and history of preoperative extracorporeal lithotripsy between groups $\mathrm{S}$ and $\mathrm{F}$. These two factors are also the indirect manifestations of stone impaction; however, the multivariate analysis demonstrated no statistically significant difference. There may be more interference effects toward these two factors; for example, some cases with early stone impaction may not have obvious hydronephrosis, and various lithotrite machines in vitro cannot evaluate the extracorporeal lithotripsy. El-Hefnawy et al. [25] reported that the degree of hydronephrosis is irrelevant to the lithotripsy failure rate, but El-Nahas et al. [26] found that the failure rate of cases of mild hydronephrosis was $13.7 \%(83 / 607)$ and that of cases of moderate to severe hydronephrosis was $24.6 \%$ (74/301) ( $p<0.01)$, but the multivariate analysis also showed no statistically significant difference. Fuganti et al. [27] reported that the history of extracorporeal lithotripsy is an important risk factor for ureteroscopy failure.

Stone site is also an important factor affecting surgical success rate. The results of this study showed that the surgical failure rate in the upper ureter was 19.7\% (18/91), significantly higher than that in the middle and lower segments (9.9\%; 29/294). The stones in the upper ureter may move to the kidneys during surgery, which is the largest factor affecting the success rate. The ESWL is the first choice for the treatment of upper ureteral calculi, but the effects in cases of stone impaction or impaired renal function are unsatisfactory. For upper ureteral stones, HLU has a lower rate of stone migration than pneumatic lithotripsy [28]. For patients at risk of stone migration, an indwelling double J tube can be placed, followed by ESWL or direct transfer to ureteral flexible lithotripsy during surgery [19]. In this study, 10 cases were successfully directly transferred to ureteral flexible lithotripsy intraoperatively. Moreover, the holmium laser induces less damage to the ureter; when combined with ureteroscopy, it can make minimally invasive treatment more efficient and safer for urinary calculi [29]. Bagley et al. [30] preferred to use flexible HLU for all upper ureteral stones. The use of stone-blocking baskets during surgery can significantly reduce the risk of intraoperative stone migration [31].

The main reasons for ureteral lithotripsy failure in the middle and lower ureter are concomitant ureteral diseases such as distal ureteral stenosis and/ or distortion, which may prevent ureteral ureteroscope placement and obstruct its upward extension. In this group, 18 cases failed due to ureteroscope placement difficulty. Therefore, surgeons should master certain techniques and strictly select the ureteroscopic indications. If the conditions allow, smaller-diameter ureteroscopes can be selected to help improve the success rate. The placement procedure should be stopped if difficulties are encountered to prevent increasing the risk of perforation, mucosal avulsion, or other ureteral injuries. Post-catheterization extracorporeal lithotripsy or open/percuta- 
neous nephrolithotomy may be selected; in cases of severe hydronephrosis in the upper and lower ureter, percutaneous nephrostomy can be considered first. After the hydronephrosis is decreased and ureteral expansion, circuitry, and angulation improve, related surgery can be performed.

This study also has some limitations. First, this was a single-center retrospective analysis, and the success rates in different periods may differ, which may have impacted certain results. Less invasive multi-center lateral studies performed in a short period together with an enlarged sample size may have better results. In addition, during clinical observations, we also found that ureteroscopy is more difficult to perform in obese patients than in nonobese patients due to a higher risk of ureteral injury. Meanwhile, stone hardness and surgeon experience also influence the success rate. Due to the imperfection of the statistical data (missing data), the above factors were not included in the analysis. We will further refine the experimental design and expand the sample size in the future to complete the statistical analysis.

\section{Conclusions}

The HLU is a safe and effective way to treat ureteral calculi. Stone impaction and upper ureteral calculi are important risk factors for surgical failure. Patients should be properly selected preoperatively, and certain surgical skills should be well mastered before surgery to avoid relevant complications.

\section{Acknowledgments}

This study was funded by the Key Discipline Construction Projects of Xiamen Medical Center (Xiaweikejiaozhongyi (2006) No. 271).

\section{Conflict of interest}

The authors declare no conflict of interest.

\section{References}

1. Bryniarski P, Paradysz A, Zyczkowski M, et al. A randomized controlled study to analyze the safety and efficacy of percutaneous nephrolithotripsy and retrograde intrarenal surgery in the management of renal stones more than $2 \mathrm{~cm}$ in diameter. J Endourol 2012; 26: 52-7.

2. Aldoukhi AH, Roberts WW, Hall TL, Ghani KR. Holmium laser lithotripsy in the new stone age: dust or bust? Front Surg 2017; 4: 57.
3. Cui Y, Cao W, Shen H, et al. Comparison of ESWL and ureteroscopic holmium laser lithotripsy in management of ureteral stones. PLoS One 2014; 9: e87634.

4. Xu G, Wen J, Li Z, et al. A comparative study to analyze the efficacy and safety of flexible ureteroscopy combined with holmium laser lithotripsy for residual calculi after percutaneous nephrolithotripsy. Int J Clin Exp Med 2015; 8: 4501-7.

5. Abdel-Kader MS. Management of calcular anuria in adults caused by ureteric stones: by using of ureteroscopy and holmium laser. Arab J Urol 2011; 9: 179-82.

6. Preminger GM, Tiselius HG, Assimos DG, et al. 2007 Guideline for the management of ureteral calculi. J Urol 2007; 178: 2418-34.

7. Turan T, Efioglu O, Danacioglu Y, et al. Can intervals in extracorporeal shock wave lithotripsy sessions affect success in the treatment of upper ureteral stones? Videosurgery Miniinv 2018; 13: 507-11.

8. Mi J, Li J, Zhang Q, et al. Combining ultrasonography and noncontrast helical computerized tomography to evaluate Holmium laser lithotripsy. Medicine (Baltimore) 2016; 95: e5564.

9. Adanur S, Aydin HR, Ozkaya F, et al. Holmium laser lithotripsy with semi-rigid ureteroscopy: a first-choice treatment for impacted ureteral stones in children? Med Sci Monit 2014; 20: 2373-9.

10. Elhilali MM, Badaan S, Ibrahim A, Andonian S. Use of the moses technology to improve holmium laser lithotripsy outcomes: a preclinical study. J Endourol 2017; 31: 598-604.

11. Bader MJ, Pongratz T, Khoder W, et al. Impact of pulse duration on Ho: YAG laser lithotripsy: fragmentation and dusting performance. World J Urol 2015; 33: 471-7.

12. Türk C, Petřík A, Sarica K, et al. EAU guidelines on interventional treatment for urolithiasis. Eur Urol 2016; 69: 475-82.

13. Ordon M, Andonian S, Blew B, et al. CUA guideline: management of ureteral calculi. Can Urol Assoc J 2015; 9: E837-51.

14. Geavlete P, Georgescu D, Niță G, et al. Complications of 2735 retrograde semirigid ureteroscopy procedures: a single center experience. J Endourol 2006; 20: 179-85.

15. Seitz C, Tanovic E, Kikic Z, Fajkovic H. Impact of stone size, location, composition, impaction, and hydronephrosis on the efficacy of holmium: YAG-laser ureterolithotripsy. Eur Urol 2007; 52: 1751-7.

16. Khoder WY, Bader M, Sroka R, et al. Efficacy and safety of Ho:YAG laser lithotripsy for ureteroscopic removal of proximal and distal ureteral calculi. BMC Urol 2014; 14: 62.

17. Chen CS, Wu CF, Shee JJ, Lin WY. Holmium: YAG lasertripsy with semirigid ureterorenoscope for upper-ureteral stones. J Endourol 2005; 19: 780-4.

18. Sugihara T, Yasunaga $\mathrm{H}$, Horiguchi $\mathrm{H}$, et al. A nomogram predicting severe adverse events after ureteroscopic lithotripsy: 12372 patients in a Japanese national series. BJU Int 2013; 111: 459-66.

19. Tanriverdi O, Silay MS, Kadihasanoglu M, et al. Revisiting the predictive factors for intra-operative complications of rigid ureteroscopy: a 15-year experience. Urol J 2012; 9: 457-64.

20. Abdelrahim AF, Abdelmaguid A, Abuzeid H, et al. Rigid ureteroscopy for ureteral stones: factors associated with intraoperative adverse events. J Endourol 2008; 22: 277-80.

21. Johnson DB, Pearle MS. Complications of ureteroscopy. Urol Clin North Am 2004; 31: 157-71. 
22. Yaycioglu O, Guvel S, Kilinc F, et al. Results with $7.5 \mathrm{~F}$ versus $10 \mathrm{~F}$ rigid ureteroscopes in treatment of ureteral calculi. Urology 2004; 64: 643-6.

23. Jhanwar A, Bansal A, Sankhwar S, et al. Outcome analysis of holmium laser and pneumatic lithotripsy in the endoscopic management of lower ureteric calculus in pediatric patients: a prospective study. Int Braz I Urol 2016; 42: 1178-82.

24. Binbay M, Tepeler A, Singh A, et al. Evaluation of pneumatic versus holmium:YAG laser lithotripsy for impacted ureteral stones. Int Urol Nephrol 2011; 43: 989-95.

25. El-Hefnawy AS, El-Nahas AR, El-Tabey NA, et al. Bilateral same-session ureteroscopy for treatment of ureteral calculi: critical analysis of risk factors. Scand J Urol Nephrol 2011; 45: 97-101.

26. El-Nahas AR, El-Tabey NA, Eraky I, et al. Semirigid ureteroscopy for ureteral stones: a multivariate analysis of unfavorable results. J Urol 2009; 181: 1158-62.

27. Fuganti PE, Pires S, Branco R, Porto J. Predictive factors for in traoperative complications in semirigid ureteroscopy: analysis of 1235 ballistic ureterolithotripsies. Urology 2008; 72: 770-4.

28. Bapat SS, Pai KV, Purnapatre SS, et al. Comparison of holmium laser and pneumatic lithotripsy in managing upper-ureteral stones. J Endourol 2007; 21: 1425-7.

29. Aboutaleb H, Omar M, Salem S, Elshazly M. Management of upper ureteral stones exceeding $15 \mathrm{~mm}$ in diameter: shock wave lithotripsy versus semirigid ureteroscopy with holmium:yttrium-aluminum-garnet laser lithotripsy. SAGE Open Med 2016 4: 2050312116685180.

30. Bagley DH, Kuo RL, Zeltser IS. An update on ureteroscopic instrumentation for the treatment of urolithiasis. Curr Opin Uro 2004; 14: 99-106.

31. Eisner BH, Dretler SP. Use of the stone cone for prevention of calculus retropulsion during holmium: YAG laser lithotripsy: case series and review of the literature. Urol Int 2009; 82: 356-60.

Received: 3.02.2019, accepted: 17.04.2019 